\title{
Accuracy of Thoracolumbar Pedicle Screw Insertion Based on Routine Use of Intraoperative Imaging and Navigation
}

\author{
Pirateb Paramasivam Meenakshi Sundaram ${ }^{1}$, Jacob Yoong-Leong $\mathrm{Oh}^{2}$, \\ Mark Tan ${ }^{2}$, Colum Patrick Nolan ${ }^{3}$, Chun Sing Yu², Ji Min Ling ${ }^{3}$ \\ ${ }^{1}$ Lee Kong Chian School of Medicine, Nanyang Technological University, Singapore \\ ${ }^{2}$ Spine Division, Department of Orthopaedic Surgery, Tan Tock Seng Hospital, Singapore \\ ${ }^{3}$ Department of Neurosurgery, National Neuroscience Institute, Singapore
}

Study Design: Retrospective review.

Purpose: To determine the accuracy of thoracolumbar pedicle screw insertion with the routine use of three-dimensional (3D) intraoperative imaging and navigation over a large series of screws in an Asian population.

Overview of Literature: The use of 3D intraoperative imaging and navigation in spinal surgery is aimed at improving the accuracy of pedicle screw insertion. This study analyzed 2,240 pedicle screws inserted with the routine use of intraoperative navigation. It is one of very few studies done on an Asian population with a large series of screws.

Methods: Patients who had undergone thoracolumbar pedicle screws insertion using intraoperative imaging and navigation between 2009 and 2017 were retrospectively analyzed. Computed tomography (CT) images acquired after the insertion of pedicle screws were analyzed for breach of the pedicle wall. The pedicle screw breaches were graded according to the Gertzbein classification. The breach rate and revision rate were subsequently calculated.

Results: A total of 2,240 thoracolumbar pedicle screws inserted under the guidance of intraoperative navigation were analyzed, and the accuracy of the insertion was $97.41 \%$. The overall breach rate was $2.59 \%$, the major breach rate was $0.94 \%$, and the intraoperative screw revision rate was $0.7 \%$. There was no incidence of return to the operating theater for revision of screws.

Conclusions: The routine use of 3D navigation and intraoperative CT imaging resulted in consistently accurate pedicle screw placement. This improved the safety of spinal instrumentation and helped in avoiding revision surgery for malpositioned screws.

Keywords: Thoracic vertebrae; Lumbar vertebrae; Surgery; Intraoperative imaging; Navigation

\section{Introduction}

The advent of three-dimensional (3D) intraoperative imaging and navigation in spinal surgery aims at improv- ing the accuracy of spinal instrumentation and thereby minimize complications and enable better outcomes for patients $[1,2]$. The 3D navigation has been used as an adjunct in spinal instrumentation, and results accumulated

Received Feb 13, 2020; Revised Apr 26, 2020; Accepted Apr 26, 2020

Corresponding author: Ji Min Ling

Department of Neurosurgery, National Neuroscience Institute, 11 Jalan Tan Tock Seng, Singapore 308433

Tel: +65-6357-7153, Fax: +65-6256-4755, E-mail: ling.ji.min@singhealth.com.sg 
over the past 10 years have demonstrated better accuracy compared with freehand and fluoroscopic techniques [35].

The use of 3D navigation in spinal instrumentation differs from one center to another, with some surgeons using it only for complex cases, whereas others using it routinely. In Tan Tock Seng Hospital, Singapore, we have adopted the routine use of $3 \mathrm{D}$ intraoperative imaging and navigation for thoracolumbar pedicle screw instrumentation.

In this study, we analyzed 2,240 pedicle screws inserted with the routine use of intraoperative navigation. Due to the differences in pedicle morphology between the Asian and Caucasian population [6], we believe that it is important to specifically analyze the accuracy of spinal instrumentation in the Asian population as well. To our knowledge, this is one of very few studies done on an Asian population with a large series of screws. This study is also a continuation of a former investigation with its preliminary data published in 2014 to reevaluate the results with a longer duration and substantially more data [7].

The aim of this study was to determine the accuracy of thoracolumbar pedicle screw insertion with the routine use of 3D intraoperative imaging and navigation over a large series of screws in an Asian population.

\section{Materials and Methods}

\section{Navigation setup and surgical techniques}

The O-arm (Medtronic, Minneapolis, MN, USA), a mobile cone beam imaging system, was used for intraoperative imaging. The $\mathrm{O}$-arm has a dual-function of both twodimensional fluoroscopy and 3D volumetric imaging, being able to produce $3 \mathrm{D}$ images with quality close to those of computed tomography.

Patients were placed in the prone position during surgery. The $\mathrm{O}$-arm machine was fit with a sterile drape and centered over the relevant levels for pedicle screw insertion. Once the spine was exposed, the reference array was clamped onto the spinous process of the vertebra one level above the uppermost instrumented vertebra to serve as a reference point for the navigation system. Subsequently, a spin of the $\mathrm{O}$-arm machine was performed to obtain $3 \mathrm{D}$ images, which is then automatically transferred to the navigation system (StealthStation S7 Navigation System; Medtronic). The surgical tools were then registered to the navigation system. The accuracy of the navigation was then verified by cross-checking the information displayed on the system with anatomical landmarks on the surgical field, such as the tip of a spinous process.

Intraoperative navigation was used to plan for the entry point, trajectory, screw length, and diameter before the insertion of pedicle screws. The desired entry point was decorticated with a high-speed drill, and the pedicle tract was created using a navigated pedicle probe, followed by navigated tapping. The pedicle screw was inserted to a suitable depth with the aid of a navigated screwdriver. The position and trajectory of the screws were observed in real-time on the monitor as it was advanced.

In patients where a minimally invasive technique was applied, the spinous process of the vertebra above the uppermost instrumented vertebra was exposed using a short midline incision for the attachment of the reference array. The location of the skin incision was marked with a navigation probe with $5-$ to $7-\mathrm{cm}$ virtual extension. After making a skin incision, a navigated Jamshidi needle was advanced through the pedicle into the vertebral body. A $\mathrm{K}$-wire was passed through the hollow needle, after which the needle was removed. Sequential dilators were placed over the K-wires, followed by tapping. A cannulated pedicle screw was inserted. An intraoperative 3D scan was performed after inserting all the screws.

\section{Data selection}

Patients who had undergone spinal surgeries involving thoracolumbar pedicle screw insertion using 3D intraoperative navigation from February 2009 to October 2017 at Tan Tock Seng Hospital, Singapore, were retrospectively reviewed. Data collected from the review included demographic details, indication for surgery, intraoperative findings, preoperative and postoperative neurological examination findings, and intraoperative 3D images. Patients who did not undergo a post-instrumentation 3D imaging or whose intraoperative 3D images were not saved in the system were excluded from the analysis.

\section{Analysis of the accuracy of screw placement}

The accuracy of the pedicle screw placement was evaluated based on intraoperative 3D images acquired by the $\mathrm{O}$-arm. The 3D axial images were analyzed for any medial or lateral breach of the pedicle wall. The pedicle breaches 
were then graded according to the Gertzbein classification (grade 0 , no breach; grade $1,<2 \mathrm{~mm}$; grade $2,2-4 \mathrm{~mm}$; grade $3,>4 \mathrm{~mm}$ ). The extent of the breach is measured in terms of the distance between the border of the pedicle wall that has been breached and the most extreme aspect of the pedicle screw medially or laterally. The breach rate of each grade and the overall breach rate were calculated. In addition, the breach rate of each individual vertebra was calculated.

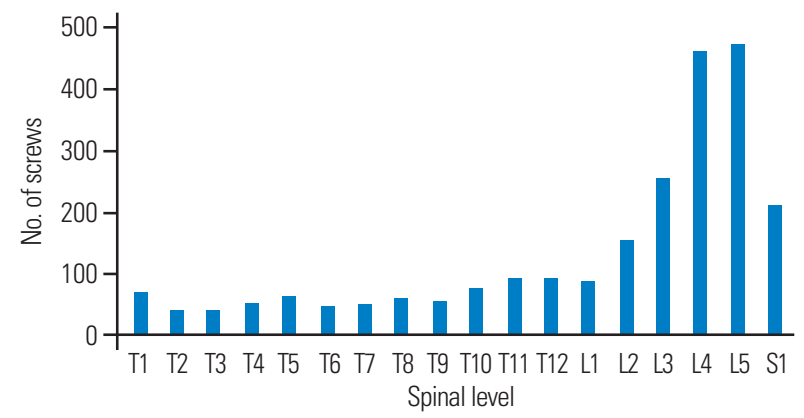

Fig. 1. Number of screws inserted at each spinal level.

Table 1. Numbers of screws inserted and breaches of individual vertebra

\begin{tabular}{lccccc} 
Level & $\begin{array}{c}\text { No. of } \\
\text { screws }\end{array}$ & $\begin{array}{c}\text { Grade 1 } \\
\text { breach }\end{array}$ & $\begin{array}{c}\text { Grade 2 } \\
\text { breach }\end{array}$ & $\begin{array}{c}\text { Grade 3 } \\
\text { breach }\end{array}$ & $\begin{array}{c}\text { Breach } \\
\text { rate (\%) }\end{array}$ \\
\hline T1 & 59 & 0 & 0 & 0 & 0 \\
\hline T2 & 28 & 0 & 0 & 1 & 3.57 \\
\hline T3 & 29 & 1 & 0 & 0 & 3.45 \\
\hline T4 & 45 & 3 & 0 & 0 & 6.67 \\
\hline T5 & 55 & 1 & 0 & 0 & 1.82 \\
T6 & 40 & 2 & 0 & 0 & 5.00 \\
\hline T7 & 42 & 0 & 2 & 0 & 4.76 \\
\hline T8 & 51 & 1 & 2 & 0 & 5.88 \\
\hline T9 & 46 & 0 & 4 & 0 & 8.70 \\
\hline T10 & 68 & 0 & 0 & 0 & 0.00 \\
\hline T11 & 85 & 2 & 0 & 0 & 2.35 \\
\hline T12 & 87 & 0 & 0 & 0 & 0 \\
\hline L1 & 79 & 1 & 0 & 0 & 1.27 \\
\hline L2 & 146 & 3 & 0 & 0 & 2.05 \\
\hline L3 & 250 & 4 & 0 & 2 & 2.40 \\
L4 & 456 & 17 & 3 & 4 & 5.26 \\
\hline L5 & 470 & 1 & 3 & 0 & 0.85 \\
\hline S1 & 204 & 1 & 0 & 0 & 0.49 \\
\hline Total & 2,240 & 37 & 14 & 7 & 2.59 \\
\hline & & & & & \\
\hline
\end{tabular}

\section{Ethics approval}

This research was approved by the Singapore National Healthcare Group Domain Specific Review Board (approval reference no., 2018/00732). Written informed consent is not necessary for patients with approval of the study by the ethics board of our institution.

\section{Results}

We analyzed a total of 2,240 thoracolumbar pedicle screws inserted under the guidance of intraoperative navigation. Fig. 1 shows the number of screws inserted at each level, and their respective breach rates are presented in Table 1 and Fig. 2. The number of breaches and intraoperative revision surgeries for the various grades of breach are shown in Table 2. The accuracy of thoracolumbar pedicle screw insertion was $97.41 \%$ (grade 0 ) in the first pass. The overall breach rate was $2.59 \%$ ( 58 of 2,240 screws). In total, 37,14 , and seven screws were considered to be grades 1,2 , and 3 breaches, respectively. Grades 2 and 3 breaches were considered to be major, with the major breach rate being $0.94 \%$. Eleven of grade 2 breaches and five of grade 3 breaches were revised intraoperatively, resulting in an intraoperative screw revision rate of $0.7 \%$. Fig. 3 exemplifies the intraoperative images demonstrating a grade 1 breach with no intraoperative revision performed and

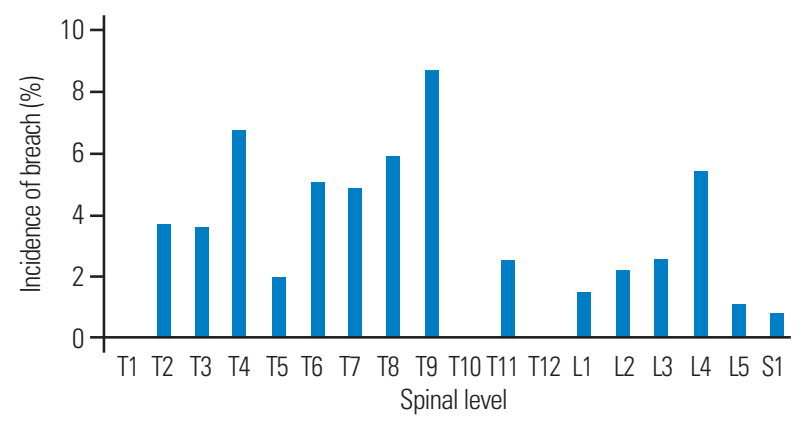

Fig. 2. Incidence of pedicle screw breach at each spinal level.

Table 2. Breach rate and revision rate of grades $1-3$ breaches

\begin{tabular}{lcccc} 
Grade of breach & $\begin{array}{c}\text { No. of } \\
\text { breaches }\end{array}$ & $\begin{array}{c}\text { Intraoperative } \\
\text { revisions }\end{array}$ & $\begin{array}{c}\text { Breach } \\
\text { rate (\%) }\end{array}$ & $\begin{array}{c}\text { Revision } \\
\text { rate (\%) }\end{array}$ \\
\hline 1 & 37 & 0 & 1.65 & 0 \\
\hline 2 & 14 & 11 & 0.63 & 0.5 \\
\hline 3 & 7 & 5 & 0.31 & 0.2 \\
\hline Overall & 58 & 16 & 2.59 & 0.7 \\
\hline
\end{tabular}



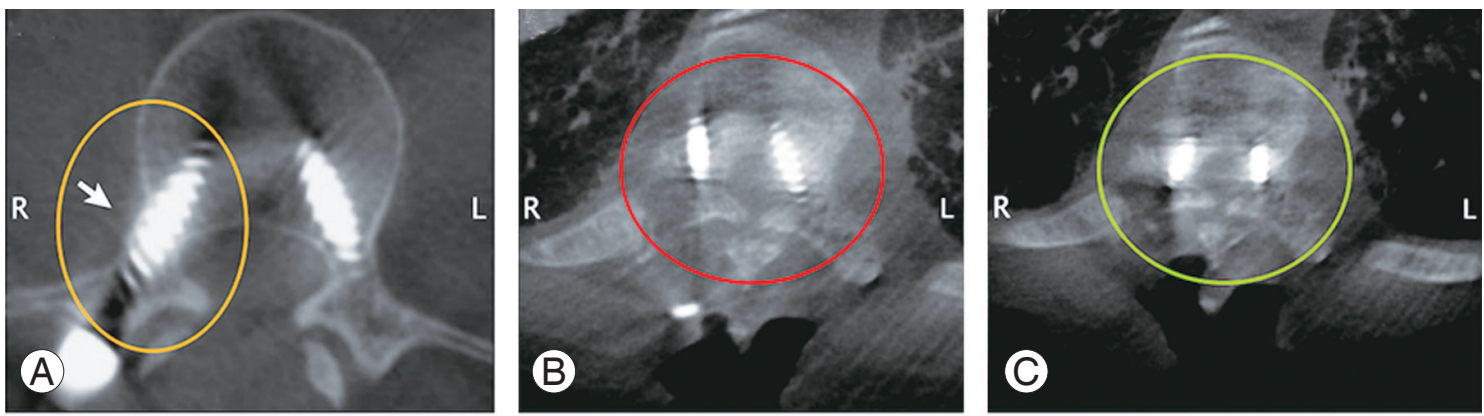

Fig. 3. (A) Grade 1 lateral breach of right pedicle screw. (B) Grade 2 lateral breach of right pedicle screw and grade 2 medial breach of left pedicle screw-pre-revision. (C) Breaches shown in Fig. 3B post-revision.

\begin{tabular}{|c|c|}
\hline Direction of breach & No. of breach (\%) \\
\hline \multicolumn{2}{|l|}{ Medial breach } \\
\hline Grade 1 & 12 \\
\hline Grade 2 & 7 \\
\hline Grade 3 & 0 \\
\hline Sub-total & $19(33)$ \\
\hline \multicolumn{2}{|l|}{ Lateral breach } \\
\hline Grade 1 & 25 \\
\hline Grade 2 & 7 \\
\hline Grade 3 & 7 \\
\hline Sub-total & $39(67)$ \\
\hline Total & $58(100)$ \\
\hline
\end{tabular}

a grade 2 breach pre- and post-intraoperative revision. There was no incidence of return to the operating theater for revision surgery related to screw malposition. There were more lateral breaches (66\%) than medial breaches (33\%). Table 3 shows a detailed breakdown according to the grade of the breach.

\section{Discussion}

As demonstrated in our study, which involved multiple surgeons with different years of experience, the use of spinal navigation resulted in a homogeneously high level of accuracy of pedicle screw placement. We observed that $97.4 \%$ of the screws in our series were successfully implanted in the first pass, which provided the best pull-out strength compared with screws that require revision. Over the past decade, spinal navigation has been more widely adopted for several reasons. Navigation offers multiple benefits such as greater accuracy, enhanced optimal fixa- tion, and increased safety $[2,8,9]$. It also provides greater confidence in case of screws of a larger diameter and length to be inserted in a more medial trajectory $[7,10]$, thereby providing optimal fixation in complex cases such as scoliosis, malignancy, osteoporosis, and deformity correction [11]. Furthermore, navigation provides increased safety with a reduction in the occurrence of neurological injuries as reported in the literature [8]. In the lumbar region where the pedicles are wider, a more medially angulated screw could be planned with navigation, thus enhancing its pull-out strength. The addition of an intraoperative O-arm check scan also ensures that no patient needs to return to the operating theater for malpositioned screw(s). Therefore, the primary advantage of intraoperative navigation is the safe and accurate screw insertion on the first attempt itself, thereby reducing the risk of a pedicle breach or an injury to the nearby neural or vascular structures, as well as the need for screw revision [7,12-14].

The accuracy obtained in this study is comparable to that of other studies. A similar study conducted by Waschke et al. [15] analyzed 2,422 screws and obtained a $94.5 \%$ accuracy with no reported revision surgeries. Another study performed by Rivkin and Yocom [16] involving 1,651 screws reported a $94.7 \%$ accuracy with a $1.5 \%$ revision surgery rate. In addition, van de Kelft et al. [5] analyzed 1,922 screws and obtained an accuracy rate of $97.5 \%$ with a $1.8 \%$ screw revision rate and no reported revision surgeries. A meta-analysis performed by Tian and $\mathrm{Xu}$ [17] analyzing more than 7,500 screws inserted with navigation reported an accuracy close to $91 \%$, and another study done by Gelalis et al. [2] analyzing 1,635 screws reported an accuracy of $89 \%-100 \%$.

In comparison, the accuracy of freehand technique reported in the literature straddled across a wide range from $71.9 \%$ to $98.3 \%$ [18-21], the difference of which is 
likely due to the heterogeneity of case complexity and the surgeon's experience. The use of freehand technique also has a significant learning curve as it was observed that the accuracy increased when compared across the period of study [18]. Meanwhile, the accuracy of the fluoroscopy technique reported in the literature ranges from $57.0 \%$ to 92.2\% [10,22-24]. These data indicate that intraoperative navigation provides greater accuracy in pedicle screw insertion than freehand technique and fluoroscopy.

A recent study conducted by Soh et al. [6] on pedicle morphology and pedicle width size in an Asian population found the narrowest pedicles to be located in the mid-thoracic region from $\mathrm{T} 3$ to $\mathrm{T} 9$. We found a certain degree of correlation between this and the breach rates in our study, with an increased rate of breach in the midthoracic region as shown in Table 1. The study conducted by Soh et al. [6] also reported that the narrowest pedicles were of the T4 $(2.9 \pm 1 \mathrm{~mm})$ and $\mathrm{T} 5(3.1 \pm 1.1 \mathrm{~mm})$ vertebrae. However, our breach rates do not correlate perfectly with this, with breaches in T9 vertebra found to be the most common in our study with a breach rate of $8.7 \%$. On the other hand, breach rates of the $\mathrm{T} 4$ and $\mathrm{T} 5$ vertebrae were $6.67 \%$ and $1.82 \%$, respectively. However, there is a need to analyze a greater number of screws in the midthoracic region to show a more reliable trend. Regarding the analysis of the direction of screw perforation, our study revealed a greater number of lateral breaches than medial breaches, with the majority of lateral breaches being of grade 1, which could be due to an "in-out-in" configuration planned by the surgeon, as well as the surgeon aiming the screw more laterally to prevent a medial breach and thus avoid the risk of spinal cord injury.

Despite the benefits of intraoperative navigation, potential drawbacks do exist. The routine use of intraoperative navigation remains contested in some centers as it has not shown to add significant value for simple cases $[8,25,26]$. It also leads to increased operative time and involves added costs to patients and healthcare systems. Furthermore, the use of navigation involves a steep learning curve, and when used in only complex surgical cases, surgeons may lack the experience and technical expertise required to ensure favorable outcomes in those complex cases $[16,27,28]$. However, the routine use in both simple and complex cases can help surgeons overcome this steep learning and become more efficient with its use over time. A subanalysis of our dataset divided into three equivalent groups according to the date of instrumentation demon- strated a decrease in breach rate from $3.89 \%$ in the earliest group to $2.82 \%$ in the middle group and $1.07 \%$ in the latest group. This reflects our learning curve and reinforces that proper training is required whenever we embrace new technology. There are also a few pitfalls that could render the navigation inaccurate. The navigation reference array has to be attached to a fixed point throughout the surgery and any deviation of the reference array could result in a navigational error. As the accuracy of the navigation system is crucial, it has to be verified after image acquisition and automatic registration of surgical tools, and precautions should be taken to not dislodge it during surgery. Any manipulation of the patient should be done with great care to ensure no movement of the vertebra at the level of instrumentation in relation to the reference array. In the case of any movement or deviation of the reference array, the reference array should be repositioned and the imaging repeated. There is also a possibility of incorrect registration of tools or error in selecting the length of the instrument or extension on the computer system. Moreover, we have observed that the imaging of the $\mathrm{C} 7$ and $\mathrm{T} 1$ levels has a poorer resolution, more so with obesity. However, the images are generally still sufficiently clear to guide the instrumentation. Therefore, we feel that surgeons who wish to include spinal navigation as a part of their surgical armamentarium should use it regularly to keep up with the skill of using navigation, not just for the surgeons but also importantly for the supporting staff such as scrub nurses and radiographers.

In addition to the numerous benefits of navigation, we found it to be a good teaching tool in a recent study performed at our institution [29]. It allows surgeons in training to correlate the surface anatomy to the internal bony anatomy. Navigation enables them to "see through" the surface landmarks. It is not a substitute to a sound understanding of anatomy. It enhances the learning of the anatomy. It gives beginners the confidence to advance the pedicle finder and tap and insert the pedicle screws. The experience in placing a pedicle screw with navigation subsequently serves as a foundation to learning the freehand technique. Trainees transition from looking at the navigation screen to paying attention to the trajectory of their instrument relative to the surface anatomy, and they also have the privilege of cross-checking with the navigation monitor when in doubt. Navigation allows the learning of a complex skill without compromising patient's safety. The end result is a spinal surgeon who is capable of both free- 
hand and navigated instrumentation.

A few limitations exist in our study. Although the intraoperative $\mathrm{O}$-arm scans consisted of $3 \mathrm{D}$ views, namely axial, sagittal, and coronal, only the axial views were uploaded to the picture archiving and communication system due to constraint of space. Therefore, only the axial images were available for analysis in our study. Therefore, it is possible that some superior or inferior breaches might not be detectable on the axial views. Second, some surgeons who were confident with their instrumentation did not repeat a post-instrumentation $\mathrm{O}$-arm scan (to reduce patient's exposure to radiation). Therefore, some cases of navigated pedicle screws were excluded from our study due to the absence of a post-instrumented scan. Third, the outcomes were not compared between different types of pathology and surgical techniques (open versus minimally invasive technique), which may affect breach rates. These data will be collected in future studies for analysis.

To summarize, our study demonstrated that the routine use of navigation and intraoperative $3 \mathrm{D}$ scanning for thoracolumbar spinal instrumentation was effective in improving safety. There was no significant neurovascular injury due to screw malposition in our series, and the few screws with significant breaches were revised during the index surgery, thereby avoiding a revision surgery for screw malposition.

\section{Conclusions}

Our analysis of a series of 2,240 thoracolumbar pedicle screw insertions revealed that spinal instrumentation using 3D intraoperative imaging and navigation allows for accurate and safe placement of pedicle screws. We observed a $97.41 \%$ accuracy of screw placement, a major breach rate of $0.93 \%$, and an intraoperative screw revision rate of $0.7 \%$. There was no incidence of return to the operating theater for revision surgery. The $3 \mathrm{D}$ intraoperative imaging and navigation can be considered as a safe and effective adjunct for spinal instrumentation particularly for complex cases.

\section{Conflict of Interest}

No potential conflict of interest relevant to this article was reported.

\section{Author Contributions}

All authors were involved in the data collection, analysis, writing and reviewing of the manuscript.

\section{References}

1. Tian NF, Huang QS, Zhou P, et al. Pedicle screw insertion accuracy with different assisted methods: a systematic review and meta-analysis of comparative studies. Eur Spine J 2011;20:846-59.

2. Gelalis ID, Paschos NK, Pakos EE, et al. Accuracy of pedicle screw placement: a systematic review of prospective in vivo studies comparing free hand, fluoroscopy guidance and navigation techniques. Eur Spine J 2012;21:247-55.

3. Larson AN, Santos ER, Polly DW Jr, et al. Pediatric pedicle screw placement using intraoperative computed tomography and 3-dimensional image-guided navigation. Spine (Phila Pa 1976) 2012;37:E188-94.

4. Oertel MF, Hobart J, Stein M, Schreiber V, Scharbrodt W. Clinical and methodological precision of spinal navigation assisted by $3 \mathrm{D}$ intraoperative $\mathrm{O}$-arm radiographic imaging. J Neurosurg Spine 2011;14:5326.

5. Van de Kelft E, Costa F, van der Planken D, Schils F. A prospective multicenter registry on the accuracy of pedicle screw placement in the thoracic, lumbar, and sacral levels with the use of the O-arm imaging system and StealthStation Navigation. Spine (Phila Pa 1976) 2012;37:E1580-7.

6. Soh TL, Kho KC, Lim ZK, Tandon AA, KaliyaPerumal AK, Oh JY. Morphological parameters of the thoracic pedicle in an Asian population: a magnetic resonance imaging-based study of 3324 pedicles. Glob Spine J 2020 Feb 24 [Epub]. https:// doi.org/10.1177/2192568220906137.

7. Ling JM, Dinesh SK, Pang BC, et al. Routine spinal navigation for thoraco-lumbar pedicle screw insertion using the $\mathrm{O}$-arm three-dimensional imaging system improves placement accuracy. J Clin Neurosci 2014;21:493-8.

8. Verma R, Krishan S, Haendlmayer K, Mohsen A. Functional outcome of computer-assisted spinal pedicle screw placement: a systematic review and metaanalysis of 23 studies including 5,992 pedicle screws. Eur Spine J 2010;19:370-5. 
9. Verma SK, Singh PK, Agrawal D, et al. O-arm with navigation versus $\mathrm{C}$-arm: a review of screw placement over 3 years at a major trauma center. Br J Neurosurg 2016;30:658-61.

10. Amato V, Giannachi L, Irace C, Corona C. Accuracy of pedicle screw placement in the lumbosacral spine using conventional technique: computed tomography postoperative assessment in 102 consecutive patients. J Neurosurg Spine 2010;12:306-13.

11. Kochanski RB, Lombardi JM, Laratta JL, Lehman RA, O'Toole JE. Image-guided navigation and robotics in spine surgery. Neurosurgery 2019;84:1179-89.

12. Watkins RG, Gupta A, Watkins RG. Cost-effectiveness of image-guided spine surgery. Open Orthop J 2010;4:228-33.

13. Xiao R, Miller JA, Sabharwal NC, et al. Clinical outcomes following spinal fusion using an intraoperative computed tomographic 3D imaging system. J Neurosurg Spine 2017;26:628-37.

14. Staartjes VE, Klukowska AM, Schroder ML. Pedicle screw revision in robot-guided, navigated, and freehand thoracolumbar instrumentation: a systematic review and meta-analysis. World Neurosurg 2018;116:433-43.

15. Waschke A, Walter J, Duenisch P, Reichart R, Kalff R, Ewald C. CT-navigation versus fluoroscopy-guided placement of pedicle screws at the thoracolumbar spine: single center experience of 4,500 screws. Eur Spine J 2013;22:654-60.

16. Rivkin MA, Yocom SS. Thoracolumbar instrumentation with CT-guided navigation (O-arm) in 270 consecutive patients: accuracy rates and lessons learned. Neurosurg Focus 2014;36:E7.

17. Tian NF, Xu HZ. Image-guided pedicle screw insertion accuracy: a meta-analysis. Int Orthop 2009;33:895903.

18. Gertzbein SD, Robbins SE. Accuracy of pedicular screw placement in vivo. Spine (Phila $\mathrm{Pa} 1976$ ) 1990;15:11-4.

19. Karapinar L, Erel N, Ozturk H, Altay T, Kaya A. Pedicle screw placement with a free hand technique in thoracolumbar spine: is it safe? J Spinal Disord Tech 2008;21:63-7.
20. Parker SL, McGirt MJ, Farber SH, et al. Accuracy of free-hand pedicle screws in the thoracic and lumbar spine: analysis of 6816 consecutive screws. Neurosurgery 2011;68:170-8.

21. Schizas C, Theumann N, Kosmopoulos V. Inserting pedicle screws in the upper thoracic spine without the use of fluoroscopy or image guidance. Is it safe? Eur Spine J 2007;16:625-9.

22. Belmont PJ Jr, Klemme WR, Dhawan A, Polly DW Jr. In vivo accuracy of thoracic pedicle screws. Spine (Phila Pa 1976) 2001;26:2340-6.

23. Carbone JJ, Tortolani PJ, Quartararo LG. Fluoroscopically assisted pedicle screw fixation for thoracic and thoracolumbar injuries: technique and short-term complications. Spine (Phila Pa 1976) 2003;28:91-7.

24. Vougioukas VI, Weber J, Scheufler KM. Clinical and radiological results after parapedicular screw fixation of the thoracic spine. J Neurosurg Spine 2005;3:2837.

25. Urbanski W, Jurasz W, Wolanczyk M, et al. Increased radiation but no benefits in pedicle screw accuracy with navigation versus a freehand technique in scoliosis surgery. Clin Orthop Relat Res 2018;476:10207.

26. Hartl R, Lam KS, Wang J, Korge A, Kandziora F, Audige $\mathrm{L}$. Worldwide survey on the use of navigation in spine surgery. World Neurosurg 2013;79:162-72.

27. Wood MJ, McMillen J. The surgical learning curve and accuracy of minimally invasive lumbar pedicle screw placement using CT based computer-assisted navigation plus continuous electromyography monitoring: a retrospective review of 627 screws in 150 patients. Int J Spine Surg 2014;8:27.

28. Hecht N, Kamphuis M, Czabanka M, et al. Accuracy and workflow of navigated spinal instrumentation with the mobile AIRO (R) CT scanner. Eur Spine J 2016;25:716-23.

29. Kaliya-Perumal AK, Soh T, Tan M, Nolan CP, Yu CS, Oh JY. Spinal navigation during orthopedic residency training: a double-edged sword? Clin Orthop Surg 2019;11:170-5. 